\title{
Stress Analysis of Full-length Bond Bolt Under Shear Deformation of Anchor Interface
}

\author{
ZHU Zhengde \\ Hohai University \\ SHU Xiaoyun \\ Hohai University \\ TIAN Yun ( $\nabla$ tianyun_2016@163.com ) \\ Shaoxing University
}

\section{Research Article}

Keywords: Full-length bond bolt, contact interface, surrounding rock deformation, the distribution of interface shear stress

Posted Date: November 30th, 2021

DOI: https://doi.org/10.21203/rs.3.rs-1106344/v1

License: (c) (i) This work is licensed under a Creative Commons Attribution 4.0 International License. Read Full License 


\title{
Stress Analysis of Full-length bond bolt under Shear Deformation of Anchor Interface
}

\author{
ZHU Zhengde ${ }^{1}$,SHU Xiaoyun ${ }^{1,2}$, TIAN Yun ${ }^{3}$ \\ (1. College of Civil and Transportation Engineering, Hohai University, Nanjing, Jiangsu 210024, China; 2. State Key \\ Laboratory of Geomechanics and Geotechnical Engineering, Institute of Rock and Soil Mechanics, Chinese Academy of Sciences, \\ Wuhan, Hubei 430071, China; 3. Key Laboratory of Rock Mechanics and Geohazards of Zhejiang Province, Shaoxing University,
}

Shaoxing 312000, China)

\begin{abstract}
Bolt anchoring force is closely related to the shear properties of the anchor interface. Considering the shear properties of anchoring agent and contact interface bonding behavior, the shear stress distribution of full-length bond bolt is analyzed based on the stress-strain relationship among bolt, anchoring agent, surrounding rock and bond interface. In this case, both the interface shear stress of the anchoring agent, surrounding rock and the bolt axial force is obtained respectively under drawing and actual working conditions. The results show that the peak shear stress of the interface, including the shear deformation of the contact interface, is significantly lower than that without it when the drawing force is applied. When designing the bolt parameters under the actual working conditions of grade IV and V surrounding rock, the relative deformation between surrounding rock and anchor should be considered, and the distribution of shear stress changes from "unimodal" to "bimodal". In the case of a lower elastic modulus of surrounding rock, both the shear stress concentration and distribution range are obvious, and the position of the neutral point is near the orifice. With the increase of elastic modulus, both the shear stress concentration and distribution range are reduced, and the position of the neutral point moves towards the depth of bolt. As a result, the optimum bolt length of full-length bond bolt can be determined by field test and decreases with the increase of elastic modulus of surrounding rock.
\end{abstract}

Keywords: Full-length bond bolt; contact interface; surrounding rock deformation; the distribution of interface shear stress

\section{Introduction}

Bolt support technology has achieved remarkable progress in the safety control of underground engineering, slope engineering in mining, water conservancy and transportation industries. Bolt support is achieved by combining the surrounding rock, anchoring agent and bolt, and the contact interface between bolt and anchoring agent -- the first interface and the contact interface between anchoring agent and surrounding rock -- the second interface. The stress transfer behavior between the two interfaces of these three materials is the key to studying the stress characteristics of the bolt. The mechanical characteristics of a full-length bond bolt are summarized below.

You [1-2] obtained the distribution of interface shear stress and axial force of bolt according to the Kelvin displacement method, and analyzed the application conditions of dispersed tension bolt. Based on that, Cao et al. [3] discussed the influence of anchoring length and proposed the support suggestions under different lithological conditions. However, without the shear characteristics of the anchorage contact interface, the solution of anchorage stress can only be obtained by assuming that the properties of the anchoring agent and surrounding rock are similar, which cannot explain the stress relationship between different interfaces of the anchoring system. Therefore, it is necessary to analyze the coordinated deformation relationship among bolt, anchoring agent and surrounding rock. Zhao [4] and He [5-6] obtained the stress distribution of the bolt based on the coordinated deformation relationship between the anchoring agent and the bolt. However, the shear properties of the interface between surrounding rock and the anchoring agent are also ignored, which fails to reflect the influence of the mechanical characteristics of the second interface. By comprehensively analyzing the stressstrain relationship between the bolt and anchoring agent, I. W. FARMER [7] obtained the distribution law of shear stress under different thicknesses of anchoring agent and proposed the calculation formula of pulling 
resistance based on indoor drawing tests. F. Delhomme [8] added the time variable in calculating the bolt drawing, which verified the significant creep behavior between the bolt and surrounding rock in the numerical simulation and laboratory experiments. However, the analysis process cannot reflect the relationship between the anchoring mechanism and the bonding interface. In addition, some scholars used mathematical models [910] to fit the experimental curve, which is highly accurate, with simple parameters, but not clear in the physical meaning, so further research is still needed.

However, there is a big difference in bolt stress between the actual working condition and the drawing test, mainly because the drawing test is difficult to reflect the influence of relative deformation between surrounding rock and anchor. Freeman [11] and Wang [18] put forward the neutral point theory, which divides the bolt length into anchor length and pick-up length under actual working conditions. The anchor length will gradually decrease due to the surrounding rock rheological properties. With the development of anchorage research, the neutral point theory has gradually become reasonable in reflecting the actual stress condition of the bolt. Based on the deformation theory of surrounding rock, $\mathrm{Li}$ [12] analyzed the interaction relationship between the full-length bond bolt and the surrounding rock. Under the condition of normal working and near failure, the analytical formula of axial force and shear stress distribution was obtained. The results show that the stress distribution of the interface conforms to the neutral point theory. TAO [13] and Tetsuro ESAKI [14] gave the formula to determine the position of the neutral point on full-length bond bolt lately. $\mathrm{Li}[17]$ took the prestress as an influencing factor to analyze the relationship of coordinated deformation between bolts and surrounding rock, with the conclusion that with the increase of prestress, the position of the neutral point is gradually transferred to the deep surrounding rock, and the shear stress decreases in the range of pick-up length, and increases in the range of anchor length. It can be seen that considering the deformation of surrounding rock is of great significance to study the actual working state of the bolt. If the anchoring agent and surrounding rock are a whole, it is difficult to reflect the shear characteristics of the second interface. Therefore, properly considering the behavior of the contact interface can help us study the mechanical characteristics of the full-length bond bolt. Considering the plastic strength of the contact interface of the anchoring agent, Cai [15] proposed the prediction method of the axial force of the bolt in soft rock engineering and concluded that the debonding failure is most likely to occur near the neutral point. By using ABAQUS finite element method, Wu [16] concluded that the deformation of surrounding rock will increase the bolt stress and transfer the peak shear stress of the interface to the depth of the bolt. It can be seen that the shear properties of the contact interfaces (the first interface and the second interface) will have an important influence on the anchoring mechanism. Establishing the shear transfer model of the two interfaces will promote the further study of the bolt stress relationship. In the actual working condition, the failure of the bolt usually occurs at the contact interface between the surrounding rock and the anchoring agent [18]. Therefore, analyzing the shear characteristics of the second interface is the key to studying the force of a full-length bond bolt.

Most previous studies found that the research of full-length bond anchorage mechanism focused on the "two materials with one interface". The bolts and the anchoring agent are assumed to be a complex whole when interface stress between the anchoring agent and surrounding rock is studied. Besides, the anchoring agent and the surrounding rock are assumed to a be complex whole when the interface stress between the bolts and the anchoring agent is studied. Nevertheless, neither of them can reflect the real mechanism of the two interfaces simultaneously. In addition, the shear stress of the bond interface is always regarded as a function of the relative rigid displacement of the two sides of the material. However, a large number of pull tests of bolts [19-20] found that a thin layer was left on the surface of the bolts or drill holes, which indicates that the anchoring bond damage is not a simple contact interface debonding failure, but a shear failure in the thin layer close to the contact interface.

In this paper, the bolt stress was analyzed with the 
consideration of the shear displacement caused by the contact interface. Based on the stress-strain relationship among bolts, anchoring agents, surrounding rock, and the two interfaces (the first interface and the second interface), the characteristics of the two interfaces were considered. The stress distribution of bolts was obtained under the actual working conditions and drawing conditions respectively, which can provide some reference for anchor design.

\section{Stress analysis of full-length bond}

\section{bolt}

\subsection{Anchorage physical model}

There is a great difference in the mechanical characteristics between the full-length bond anchorage and the end anchorage. The force transfer behavior of the anchoring agent has an important effect on the ultimate drawing force of the bolt, and its shear stress will change the shear strain of the contact interface. Under the action of drawing force, the bolt and the surrounding rock will undergo axial deformation, and the anchoring agent will undergo shear deformation. On the interface region, the shear modulus will be affected by the properties of anchoring agents. With the increment of shear deformation, the shear failure of the interface unit finally occurs, which causes the debonding failure of the interface. In this paper, the interfaces among the anchoring agent, bolt, and surrounding rock were analyzed. The basic assumptions are as follows :(1) surrounding rock, anchoring agents, and bolts are all homogeneous linear elastomers. (2) The shear stress-strain of the anchorage bond interface satisfies the linear shear model without debonding failure of the interface. (3) The influence of interfacial compressive stress on shear characteristics is not considered.

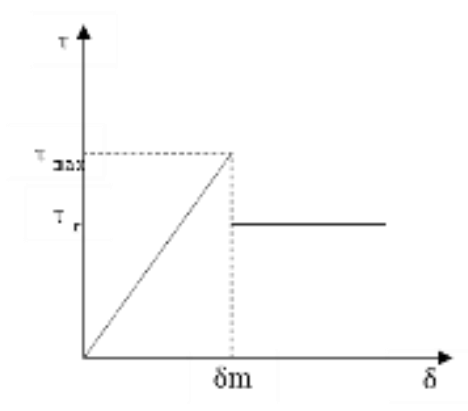

Fig. 1 Shear model of the bonded interface1

A reasonable anchorage interface shear model (including the first interface and the second interface) is the key to studying the interface mechanism among bolts, anchoring agents and surrounding rock. Based on the existing test results about the shear properties of the anchoring agent, this article assumed the mechanical properties of the anchoring interface, which is shown in Fig 1. The shear stress-strain relationship satisfies the following relation:

$$
\tau=\left\{\begin{array}{c}
\mathrm{k} \delta, 0 \leq \delta \leq \delta_{\mathrm{m}} \\
\tau_{\mathrm{r}}, \delta>\delta_{\mathrm{m}}
\end{array}\right.
$$

where $\tau$ represents the shear stress at contact interface; $\tau_{r}$ represents the residual shear stress at contact interface; $\mathrm{k}$ represents the shear modulus at the bonded contact interface; $\delta$ represents the shear strain at the contact interface; and $\delta_{m}$ represents the maximum shear strain at the contact interface.

Since the analysis in this paper is based on the elastic assumption, the residual interface strength after peak shear stress is not considered.

\subsection{Bolt stress under drawing conditions}

Under external drawing force, the elements of bolts element and anchoring agents were analyzed respectively, as shown in Fig. 2.

According to the stress balance relationship of bolt elements in Fig. 2 (a), the following equation can be obtained:

$$
\frac{\mathrm{d} \sigma}{\mathrm{d} x}=\frac{4 \tau_{1}}{\mathrm{D}_{1}}
$$

where $\sigma$ is the axial force of the bolt; $\tau_{1}$ is the shear stress at the first interface; $D_{1}$ is the diameter of the bolt, and $x$ is the distance from the bolt head. 


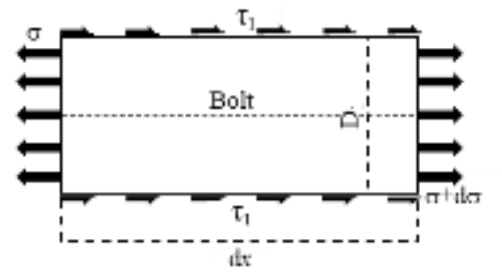

(a) Stress analysis of bolt element

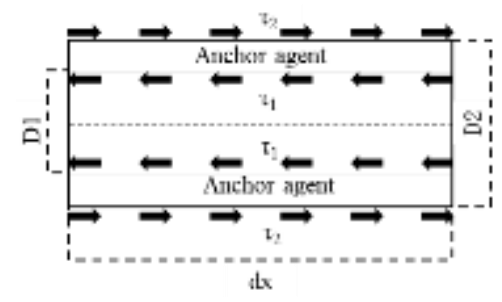

(b) Stress analysis of anchoring agent elements2

Fig.2 Stress analysis of anchor unit elements

The Anchoring agent transfers the external force from the bolt to the surrounding rock according to the shear deformation of the bolt, and the stress balance must be satisfied between the first interface and the second interface. As shown in Fig 2 (b), the following equation can be obtained:

$$
\tau_{1} \pi \mathrm{D}_{1} \mathrm{~d} x=\tau_{2} \pi \mathrm{D}_{2} \mathrm{~d} x
$$

where $D_{2}$ is the diameter of the borehole and $\tau_{2}$ is the shear stress at the second interface.

The simplified equation can be obtained as follows:

$$
\tau_{1}=\frac{\mathrm{D}_{2}}{\mathrm{D}_{1}} \tau_{2}
$$

Since $D_{2}>D_{1}, \tau_{1}$ is larger than $\tau_{2}$, which is consistent with the common knowledge that the shear stress at the second interface is caused by the attenuation of the first interface. However, a simple linear attenuation was adopted in this paper. As the shear modulus of the interface is related to the properties of the contact bodies on both sides [19], the following equation can be obtained:

$$
\mathrm{K}=\frac{\mathrm{K}_{\mathrm{a}} \mathrm{K}_{\mathrm{b}}}{\mathrm{K}_{\mathrm{a}}+\mathrm{K}_{\mathrm{b}}}
$$

where $\mathrm{K}$ is the shear modulus of interface; $\mathrm{K}_{\mathrm{a}} 、 \mathrm{~K}_{\mathrm{b}}$ are the shear modulus of the two contact bodies respectively. Since the shear modulus of the bolt is far greater than that of the surrounding rock, the shear strain generated at the first interface is relatively small. Therefore, the shear stress of the second interface (hereinafter referred to as the interface shear stress) plays an important role in maintaining the anchorage stability [18]. As shown in Fig 2 (b), the following equation can be obtained:

$$
\gamma=\frac{\tau_{2}}{\mathrm{G}_{\mathrm{b}}}
$$

where $\gamma$ is, the shear strain of anchoring agent; $\mathrm{G}_{b}$ is the shear modulus of anchoring agent.

Under the drawing condition, the coordinate deformation should be considered among the bolt, anchoring agent and surrounding rock. Without the influence of the thickness of the anchoring agent, the contact interface is regarded as a bonding element, as shown in Fig. 3.

When drawing force was applied, the shear strain of the first interface occurred because of the deformation of the bolt. According to the shear deformation of the anchoring agent, the drawing force was transferred to the second interface and surrounding rock. As shown in Fig 3, the deformation of the bolt is composed of the deformation of surrounding rock, interface and anchoring agent.

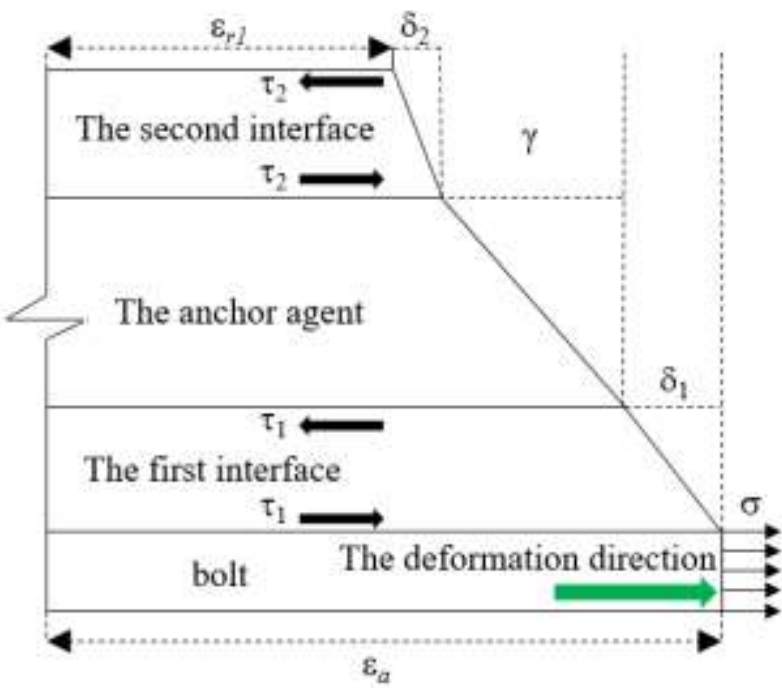

Fig.3 Strain analysis of anchorage interface under prestress 


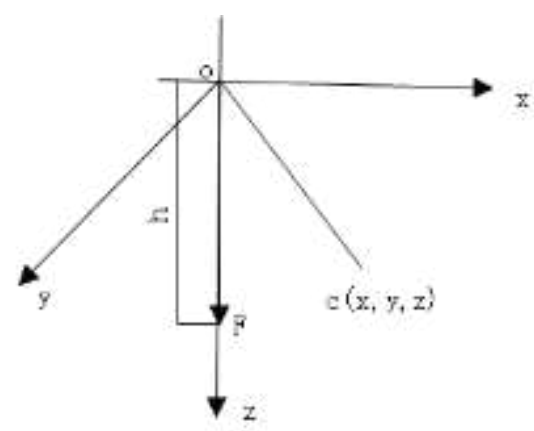

3

Fig.4 The calculation diagram of Mindlin's solution

Compared with the scale of the surrounding rock, the influence of the bolt diameter can be ignored. The rock mass influenced by the bolt can be regarded as the infinite concentrated force exerted at a depth of a halfspace region. As shown in Fig. 4, the vertical displacement at $h$ can be determined by the Mindlin displacement solution. When $\mathrm{x}=\mathrm{y}=\mathrm{z}=0$, the displacement of surrounding rock can be expressed as:

$$
\mathrm{u}_{\mathrm{r}}=\frac{\left(1+\mu_{\mathrm{r}}\right)\left(3-2 \mu_{\mathrm{r}}\right)}{2 \pi \mathrm{E}_{\mathrm{r}}} \frac{\mathrm{F}(x)}{\mathrm{h}}
$$

where $\mathrm{F}(x)$ is the axial force of the bolt at the $x$ position; $\mathrm{E}_{\mathrm{r}}$ and $\mu_{\mathrm{r}}$ are the elastic modulus and Poisson's ratio of surrounding rock, respectively.

By recombining the axial direction of the bolt with the z-axis, and according to equation (3), the strain of surrounding rock generated by the drawing force can be described as:

$$
\varepsilon_{r 1}=\frac{\mathrm{du}_{\mathrm{r}}}{\mathrm{d} x}=\frac{\mathrm{D}_{2}\left(3-2 \mu_{\mathrm{r}}\right) \tau_{2}}{4 \mathrm{G}_{\mathrm{r}} x}
$$

where $\mathrm{G}_{\mathrm{r}}$ is the shear modulus of surrounding rock.

According to hypothesis (2), the relationship between shear stress and shear strain at the contact interface of the anchoring agent is:

$$
\left\{\begin{array}{c}
\tau_{1}=\mathrm{K}_{1} \delta_{1} \\
\tau_{2}=\mathrm{K}_{2} \delta_{2}
\end{array}\right.
$$

where $K_{1}$ and $K_{2}$ are the shear modulus of the first and second interface; $\delta_{1}$ and $\delta_{2}$ are the shear strain of the first and second interface, respectively.

According to the physical equation of the bolt, the following equation can be obtained:

$$
\varepsilon_{\mathrm{a}}=-\frac{\sigma}{\mathrm{E}_{\mathrm{a}}}
$$

where $\varepsilon_{a}$ is the bolt strain; $\mathrm{E}_{\mathrm{a}}$ is the bolt elastic modulus.

As shown in Figure 3, the deformation relationship can be described as:

$$
\varepsilon_{\mathrm{a}}=\varepsilon_{\mathrm{r} 1}+\delta_{2}+\gamma+\delta_{1}
$$

Combining formula (6), (8), (9), (10) and (11), the following equation can be obtained:

$$
\left(-x^{2}+\mathrm{A}\right) \tau_{2}=\left(\mathrm{A} x+\mathrm{B} x^{2}\right) \tau_{2}^{\prime}
$$

where

$$
\begin{gathered}
A=\frac{D_{1}^{2} E_{a}\left(3-2 \mu_{r}\right)}{16 G_{r}} \\
B=\frac{\left[\left(K_{1} D_{1}+K_{2} D_{2}\right) G_{b}+K_{1} K_{2}\left(D_{2}-D_{1}\right)\right] D_{1} E_{a}}{4 G_{b} K_{1} K_{2} D_{2}}
\end{gathered}
$$

By introducing boundary conditions, we can get:

$$
\int_{0}^{L} \tau_{2} \pi D_{2} d x=\mathrm{F}
$$

where $L$ is the length of the bolt, then the solution of interface shear stress and axial force under the drawing condition can be given as:

$$
\left\{\begin{array}{l}
\tau_{2}^{(1)}=\mathrm{C}_{1} \cdot x \cdot(\mathrm{A}+\mathrm{B} x)^{\frac{\mathrm{A}}{\mathrm{B}^{2}}-1} \cdot \exp \left(-\frac{x}{\mathrm{~B}}\right) \\
F_{(x)}^{(1)}=\mathrm{C}_{1} \pi \mathrm{D}_{2} \cdot(\mathrm{B} x+\mathrm{A})^{\frac{\mathrm{A}}{\mathrm{B}^{2}}} \cdot \exp \left(-\frac{x}{\mathrm{~B}}\right)
\end{array}\right.
$$

where $C_{1}$ is the coefficient related to the boundary, which can be described as:

$$
\mathrm{C}_{1}=\frac{F}{\left[A^{\frac{A}{B^{2}}}-(A+B)^{\frac{A}{B^{2}}} \cdot \exp \left(-\frac{L}{B}\right)\right] \pi D_{2}}
$$

\subsection{Bolt stress under actual working conditions}

The internal stress of the surrounding rock can be rebalanced after tunnel excavation, which will redistribute the field of stress and displacement. Especially under the condition of soft rock, displacement can be substantial without efficient support. Therefore, the deformation of surrounding rock should be considered when designing anchorage support. The influence of surrounding rock deformation on the stress of anchor can be divided into two parts. 
The first one is the shear stress caused by the relative deformation between surrounding rock and anchoring agent. The second one is the drawing force applied by the anchor plate directly, which is related to the deformation of the tunnel surface.

2.3.1 Relative deformation of surrounding rock at the interface

With the shear characteristics of anchoring agents, the additional shear strain will occur on the second interface when surrounding rock deforms toward the tunnel. The additional shear stress will transfer to the first interface according to the shear deformation of anchoring agent. Finally, the additional force caused by the deformation of surrounding rock will be balanced by the deformation of bolt, as shown in Fig. 5.

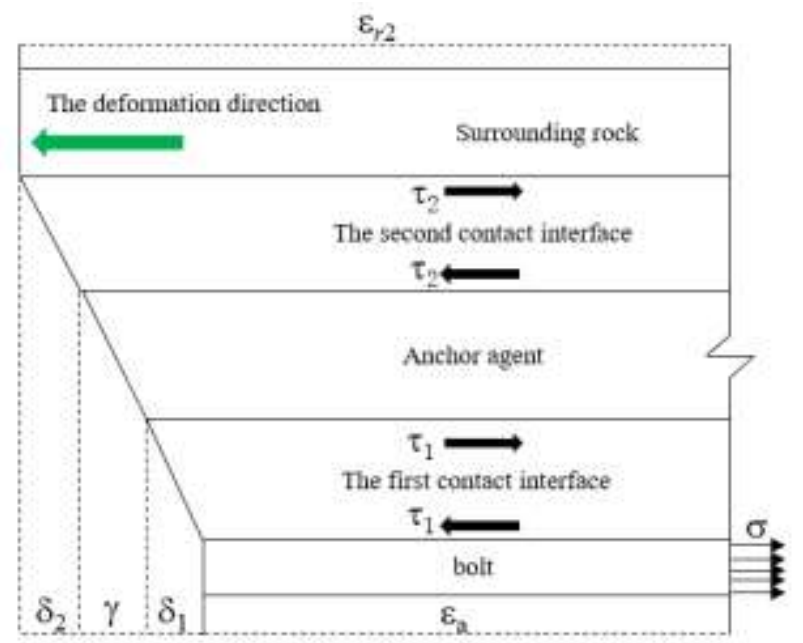

Fig.5 Strain analysis of anchorage interface under surrounding rock deformation

When the unit additional force $d N$, the following equation can be obtained:

$$
\tau_{1} \pi \mathrm{D}_{1} d x=\tau_{2} \pi \mathrm{D}_{2} d x=d N
$$

In this case, the shear strain $\delta_{2}$ at the second interface, the relative shear strain $\gamma$ at the anchoring agent, the shear strain $\delta_{1}$ at the first interface and the bolt strain $\varepsilon_{a}$ caused by the additional force can be described as:

$$
\left\{\begin{array}{c}
\delta_{2}=\frac{d N}{\pi \mathrm{D}_{2} \mathrm{~K}_{2}} \\
\gamma=\frac{\mathrm{D}_{2}-\mathrm{D}_{1}}{\mathrm{G}_{\mathrm{b}} \pi \mathrm{D}_{1} \mathrm{D}_{2}} d N \\
\delta_{1}=\frac{d N}{\pi \mathrm{D}_{1} \mathrm{~K}_{1}} \\
\varepsilon_{\mathrm{a}}=\frac{4}{\pi \mathrm{D}_{1}^{2} \mathrm{E}_{\mathrm{a}}} d N
\end{array}\right.
$$

Therefore, generated by additional unit force $d N$, the strain of surrounding rock $\varepsilon_{\Delta}$ is:

$$
\varepsilon_{\Delta}=\delta_{2}+\gamma+\delta_{1}+\varepsilon_{\mathrm{a}}
$$

Assuming that $\varepsilon_{r 2}$ is the strain of surrounding rock caused by the bolt, then the additional force of the bolt can be described as:

$$
F_{r}=\frac{d N}{\varepsilon_{\Delta}} \varepsilon_{r 2}
$$

where $F_{r}$ is the additional force of the bolt. The shear stress of the second interface can be described as:

$$
\tau_{2}=\frac{F_{r}}{\pi \mathrm{D}_{2}}=\frac{1}{\pi \mathrm{D}_{2}} \frac{d N}{\varepsilon_{\Delta}} \varepsilon_{r 2}
$$

The axial force $F_{r}$ and shear stress $\tau_{2}$ satisfy the relation:

$$
F_{r}+\int_{0}^{r} \pi \mathrm{D}_{2} \tau_{2} \mathrm{dr}=0
$$

Then the differential equation of the strain of surrounding rock under the influence of bolt is:

$$
\varepsilon_{\mathrm{r} 2}+\varepsilon_{\mathrm{r} 2}^{\prime}=0
$$

The general solution of the upper differentiation equation can be obtained as follows:

$$
\varepsilon_{\mathrm{r} 2}=\mathrm{C}_{2} \cdot \mathrm{e}^{-\mathrm{r}}
$$

Due to the lining and other constraints at the tunnel surface, the bolt head can be coordinated under the strain condition. Namely, when $r$ equals $r$, the rock strain at the tunnel surface can be expressed as

$$
\varepsilon_{r 2 \mid r 2=r_{0}}=\frac{\mathrm{P} \beta}{2 \mathrm{G}_{\mathrm{r}}}
$$

where $\beta$ is the lining influence coefficient, which can be obtained according to the elastic mechanics formula; $r_{0}$ is the radius of the tunnel, and $\mathrm{P}$ is the in-situ stress. Therefore, the strain of surrounding rock under the 
anchorage support can be described as:

$$
\varepsilon_{r 2}=\frac{\mathrm{P} \beta}{2 \mathrm{G}_{\mathrm{r}}} \cdot \mathrm{e}^{-r+r_{0}}
$$

The deformation law of surrounding rock under the influence of anchor is consistent with the result of Song [19]. Therefore, considering the deformation of surrounding rock after tunnel excavation, the distribution of interface's shear stress $\tau_{2}^{(2)}$ and bolt's axial force $F_{(x)}^{(2)}$ can be described as:

$$
\left\{\begin{array}{c}
\tau_{2}^{(2)}=\frac{1}{\pi \mathrm{D}_{2}} \cdot \frac{1}{\varepsilon_{\Delta}} \cdot \frac{\mathrm{P} \beta}{2 \mathrm{G}_{\mathrm{r}}} \cdot \mathrm{e}^{-x} \\
F_{(x)}^{(2)}=\frac{1}{\varepsilon_{\Delta}} \cdot \frac{\mathrm{P} \beta}{2 \mathrm{G}_{\mathrm{r}}} \cdot \mathrm{e}^{-x}
\end{array}\right.
$$

\subsubsection{The effect of the anchor plate}

According to hypothesis (1), the action of the anchor can be considered as a superposed process. The resilient stress reversely acts on the surrounding rock after the prestress is applied to the bolt. Thus, the displacement of the surrounding rock surface departing from the tunnel can be described as:

$$
\Delta \mathrm{U}_{1}=\int_{0}^{L} \varepsilon_{r 1} \mathrm{~d} x
$$

Considering the deformation of surrounding rock, the displacement of tunnel surface to the tunnel can be described as:

$$
\Delta \mathrm{U}_{2}=\int_{r_{0}}^{r_{0}+L} \varepsilon_{r 2} \mathrm{dr}
$$

Substituting equation (8), (26), the relative displacement between the bolt and surrounding rock under the influence of excavation and prestress can be described as:

$$
\begin{aligned}
& \Delta \mathrm{U}_{3}=-\frac{D_{2}\left(3-2 \mu_{r}\right)}{4 G_{r}} \cdot C_{1} \cdot \frac{B}{A}\left[\frac{(A+B L)^{A / B^{2}}}{A+1+B L}\right. \\
& \left.\cdot \exp \left(-\frac{L}{B}\right)-\frac{A^{A / B^{2}}}{A+1}\right]+\frac{\mathrm{P} \beta}{2 \mathrm{G}_{\mathrm{r}}} \cdot\left(1-\mathrm{e}^{-L}\right)
\end{aligned}
$$

Due to the restrain of the anchor plate, the relative displacement between the bolt and the surrounding rock will be limited. In this case, the surface of the tunnel and the bolt head should maintain the relationship of coordinate deformation, which means no relative slip between them. Therefore, the relative displacement between bolt and surrounding rock should be converted into the additional force of the bolt $\Delta F$. The following equation is obtained:

$$
\Delta F=\Delta \mathrm{U}_{3} \mathrm{E}_{\mathrm{a}} \frac{\pi \mathrm{D}_{1}^{2}}{4}
$$

According to the research of You [1], the distribution of the interface's shear stress and bolt's axial force caused by additional forces can be described as:

$$
\left\{\begin{array}{c}
\tau_{2}^{(3)}(\mathrm{x})=\frac{2 \Delta F}{\pi \mathrm{D}_{1}} \cdot\left(\frac{\mathrm{t} x}{2}\right) \exp \left(-\frac{\mathrm{t}}{2} x^{2}\right) \\
F_{(x)}^{(3)}=\Delta F \cdot \exp \left(-\frac{\mathrm{t}}{2} x^{2}\right)
\end{array}\right.
$$

where

$$
t=\frac{4}{\left(1+\mu_{r}\right)\left(3-2 \mu_{r}\right) D_{1}^{2}}\left(\frac{E_{r}}{E_{a}}\right)
$$

In this case, under the actual working condition, the stress of the anchorage can be divided into three parts:

1 The tension of the bolt departing from tunnel due to the prestress

2 The additional stress of interface pointing to the tunnel due to the deformation of the surrounding rock

3 The additional stress of the bolt pointing to the tunnel due to the deformation of the anchor plate

When the analysis object is out of prestress, the first stress can be set to zero. The stress distribution characteristics of the bolt can be described if we only consider surrounding rock deformation. Setting the direction that departs from the tunnel as the positive direction of the second interface, then the interface's shear stress and bolt's axial force under the combined influence of the three parts can be described as:

$$
\begin{aligned}
& \tau_{2}=-\mathrm{C}_{1} \cdot x \cdot(\mathrm{A}+\mathrm{Bx}) \frac{\mathrm{A}}{\mathrm{B}^{2}}-1 \cdot \exp \left(-\frac{1}{\mathrm{~B}} x\right)-\frac{1}{\pi \mathrm{D}_{2}} \cdot \frac{1}{\varepsilon_{\Delta}} \\
& \cdot \frac{\mathrm{P} \beta}{2 \mathrm{G}_{\mathrm{r}}} \cdot \mathrm{e}^{-x}+\frac{2 \mathrm{~F}}{\pi \mathrm{D}_{1}} \cdot\left(\frac{\mathrm{t} x}{2}\right) \exp \left(-\frac{\mathrm{t}}{2} x^{2}\right) \\
& F(x)=\mathrm{C}_{1} \pi \mathrm{D}_{2} \cdot(\mathrm{B} x+\mathrm{A}) \frac{\mathrm{A}}{\mathrm{B}^{2}} \cdot \exp \left(-\frac{x}{\mathrm{~B}}\right)+\frac{1}{\varepsilon_{\Delta}} \cdot \frac{\mathrm{P} \beta}{2 \mathrm{G}_{\mathrm{r}}} \cdot \mathrm{e}^{-x} \\
& -\Delta F \cdot \exp \left(-\frac{\mathrm{t}}{2} x^{2}\right)
\end{aligned}
$$


(34)

\section{Numerical simulation of full-length}

bond bolt considering deformation of

\section{the interface}

\subsection{Stress characteristics of the bolt under drawing condition}

When the bolt is subjected to drawing conditions, the stress on the bolt is caused by the overall action of the bolt, anchoring agent, surrounding rock and bonding interface. The shear properties of the interface have an important influence on the stress distribution of the bolt. Without the shear deformation of the interface, You [1] gave the traditional analytical solution of interfacial shear stress under drawing conditions. The drawing force is set to $50 \mathrm{KN}$. The diameter of the bolt is $22 \mathrm{~mm}$. The diameter of the borehole is $50 \mathrm{~mm}$, and the elastic modulus of the bolt is $210 \mathrm{GPa}$. According to the property of the existing mortar anchoring agent and the supporting conditions of the soft rock tunnel, the elastic modulus of the anchoring agent is $30 \mathrm{GPa}$, and that of the surrounding rock is $2 \mathrm{GPa}$. The length of the bolt is $4 \mathrm{~m}$, and the Poisson's ratio of all materials is 0.25 . The shear modulus of the bolt is bigger than that of the anchoring agent and surrounding rock, which will significantly enhance the shear stiffness of the first interface, resulting in that the value of $\mathrm{K} 1$ is much larger than that of K2. According to Equation (5) and reference [25], the interface parameters were set as $\mathrm{K} 1=83.6 \mathrm{GPa}$ $/ \mathrm{m}$ and $\mathrm{K} 2=9.95 \mathrm{GPa} / \mathrm{m}$. The model was established by ABAQUS, as shown in Fig. 6 (a), and the interface was simulated by using the cohesive element, as shown in Fig. 6 (b). The comparison of results among numerical simulation, the model proposed in this paper and the solution of You [1] are shown in Fig. 6 and 7.

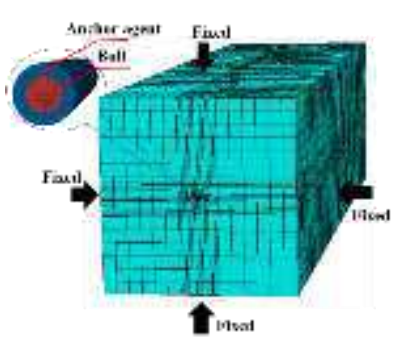

(a) Model element

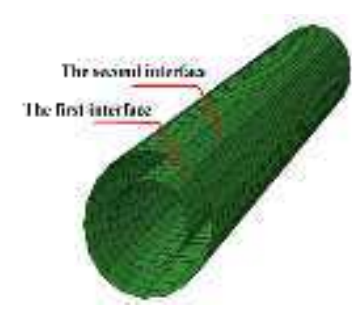

(b) interface element
Fig.6 Model schematic of tests

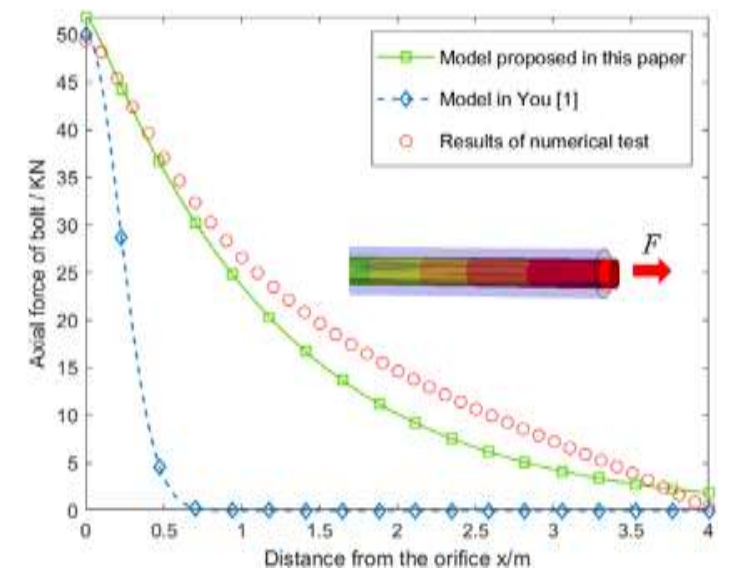

(a) Comparison of axial force under drawing condition

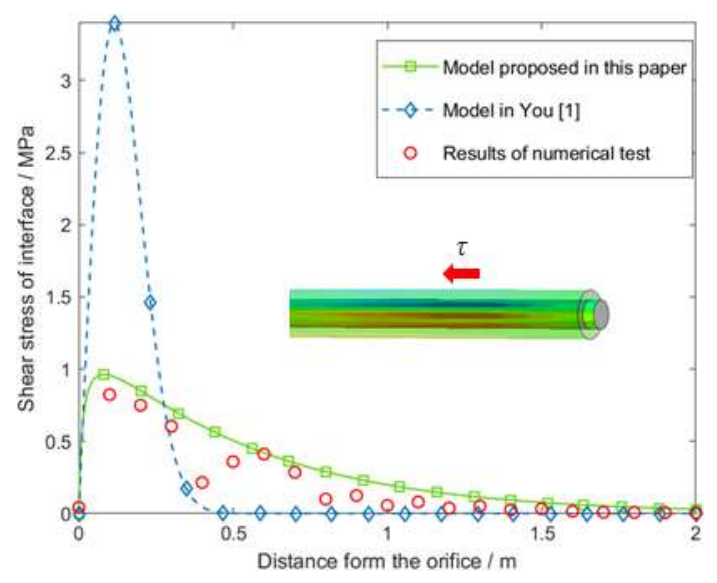

(b) Comparison of shear stress under drawing condition

Fig 7 Comparison between theoretical and numerical results of axial force and shear stress under drawing condition

It can be seen from Fig 7 that the numerical results are in good agreement with the model presented in this paper, indicating that the contact interface model based on "three materials and two interfaces" can truly reflect the internal mechanical behavior of the anchor. As the traditional calculation model excludes the shear deformation of the anchoring agent, the bolt's axial force and interface's shear stress are mainly distributed in a small range near the orifice, which is similar to the 
phenomenon that the internal stress of the rigid body is significantly concentrated when the stress is concentrated. Thus, if the shear deformation of the anchoring agent and contact interface is not considered, the degree of shear stress concentration will be significantly greater than the actual situation. When the anchoring agent is not considered as a rigid body, the interface's shear stress and the bolt's axial force will gradually transfer to the deep under the shear deformation of the anchoring agent and contact interface. As the shear modulus of the anchoring agent increases, its peak shear stress decreases while the range of interface shear stress increases significantly. However, due to the limited deformation capacity of the anchoring agent, the stress concentration near the bolt head is obvious. The region near the bolt head is the most prone to fracture and debonding failure when the drawing force is applied. Assuming the deformation of the contact interface is infinite, which means the shear modulus of the anchoring agent is zero, the interface shear stress does not participate in sharing the axial force of the bolt. In this case, the stress of the bolt shifts to the end anchorage, with the axial stress distributing along the full bolt evenly. Because the drawing force is borne by the bolt, the stress of the bolt is easier to reach the tensile strength, which leads to the destruction of the bolt.

\subsection{Stress characteristics of the bolt under actual working conditions}

Different from the drawing condition, the actual working condition of the bolt is affected by the deformation of the surrounding rock. The support of the bolt changes from passive to active, and the distribution of the interface's shear stress changes greatly. By using the numerical model of the full-length bond bolt, Wang [22] obtained the shear stress distribution at the contact interface after tunnel excavation. Ignoring the influence of lining and prestress, the first term of Equation (34) was set to zero, and the $\beta$ was equal to 1 . Then the comparison results between the model proposed in this paper and the numerical simulation of Wang [22] are shown in Fig. 9.

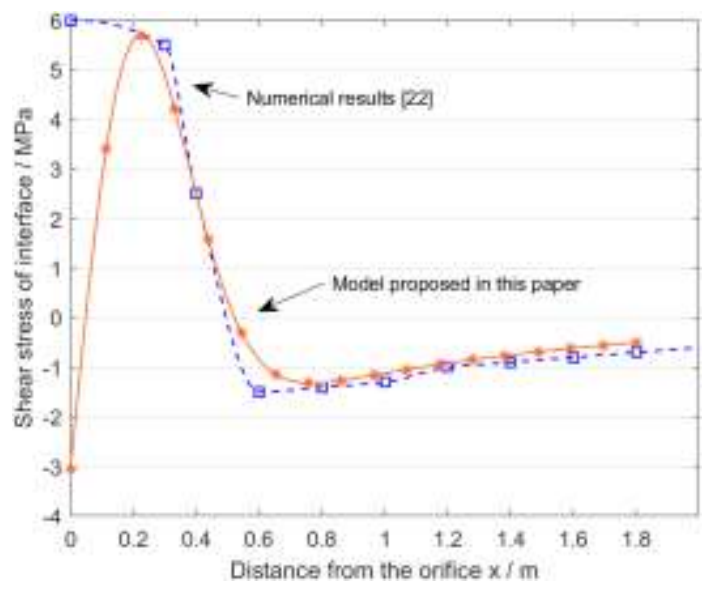

Fig 9 The verification of shear stress in the interface under actual working conditions

1) The model proposed in this paper is generally consistent with the numerical results in the literature, indicating that it can reflect the stress distribution of the bolt under the actual working condition. Due to the limitation of the anchor plate, the deformation of the surrounding rock at the orifice is coordinated and the peak point of the interface shear stress gradually moves from the orifice to the depth of the bolt. Thus, the form of interface shear stress is shifted from nonlinear attenuation into unimodal distribution.

2) Under the actual working condition, the distribution characteristics of interface shear stress prove the existence of the neutral point in the full-length bond bolt, and the position of the neutral point is closely related to the peak position of shear stress. Due to the influence of surrounding rock deformation, there is a neutral point whose shear stress is zero at the contact interface. From the distance of the bolt head to the neutral point, which is called the "pick-up length", the direction of interface shear stress points to the tunnel to limit the deformation of surrounding rock. The part from the neutral point to the bottom of the bolt, the "anchor length", the direction of interface shear stress backs to the tunnel to limit the deformation of the bolt. Thus, the neutral point becomes the demarcation point for the stress direction of the bond interface. The position of the neutral point is about $0.47 \mathrm{~m}$ away from the bolt head under this condition, which is also consistent with the results obtained in the literature [14].

3) Under the actual working condition, the distribution of interface shear stress changes from 
"single peak" to "double peak" when compared with the drawing condition, and the peak position of shear stress can be determined by field drawing tests. When the deformation of surrounding rock is considered, the shear stress of interface rapidly peaks (maximum positive shear stress) near the orifice, and the peak position is consistent with that under the drawing condition. After reverting at the neutral point, the interface shear stress attenuates rapidly to the second peak (maximum negative shear stress), but the magnitude is much smaller than the first one, which means the shear failure caused by interface is more likely to occur in the pick-up length. In this case, the peak position of interface shear stress can be found by field drawing tests when designing the bolt, and the bond strength of pick-up length should be enhanced to maintain anchorage stability.

\section{Influence of surrounding rock}

\section{properties on bolt stress characteristics}

\subsection{Influence of surrounding rock on bolt stress} under drawing condition

Due to the variability of surrounding rock conditions, the properties of surrounding rock should be fully considered when designing the anchorage parameters. The shear properties of the anchoring agent were ignored in most traditional methods, which can't reflect the stress of the bolt. The properties of the anchoring agent and contact interface will have an important impact on the stress of the bolt. Therefore, under different elastic modulus of surrounding rock, it is necessary to compare the mechanical characteristics of the bolt with and without considering the shear deformation of the interface.

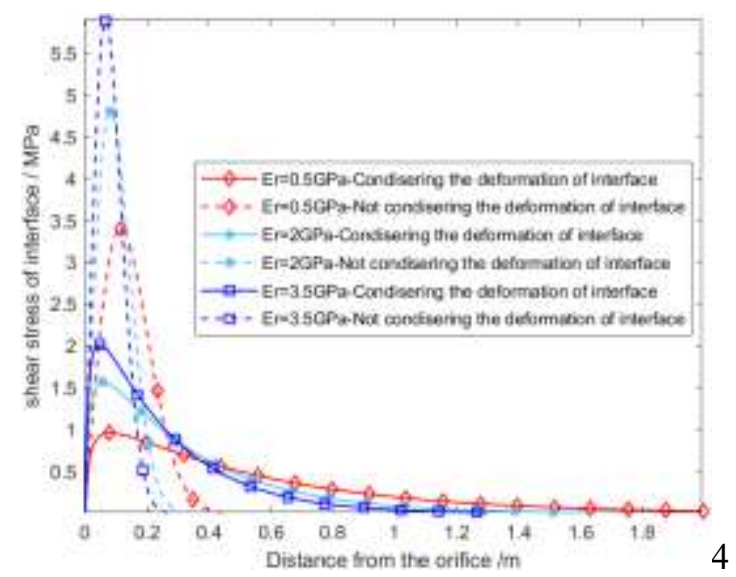

Fig 8 The influence of lithological conditions on shear stress

As shown in Fig 8, the following conclusions can be drawn:

1) Under the same elastic condition, the distribution law of interface shear stress is basically the same whether the deformation of the anchorage interface is considered or not. The shear stress presents an obvious unimodal distribution in the $1 / 3$ region of the bolt front, and decreases rapidly after reaching the shear stress peak. With the increase of the elastic modulus of the surrounding rock, the peak values of both shear stress gradually rise, and the peak position slowly moves towards the orifice.

2) The shear deformation of the contact interface can significantly affect the distribution range of shear stress and reduce the peak shear stress. When the elastic modulus of surrounding rock is $0.5 \mathrm{GPa}, 2 \mathrm{GPa}$ and 3.5 $\mathrm{GPa}$, the distribution range of interface shear stress is $0.46 \mathrm{~m}, 0.33 \mathrm{~m}$ and $0.25 \mathrm{~m}$ without considering the shear deformation of the contact interface, while the distribution range is $2.5 \mathrm{~m}, 1.8 \mathrm{~m}$ and $1.4 \mathrm{~m}$ when the shear deformation of contact interface is considered. The distribution range of shear stress increases, and the peak shear stress decreases significantly.

3) The anchoring parameters can be designed more reasonably if the influence of the anchoring agent and shear deformation of the contact interface is considered. When the bolt is subjected to drawing force, both the anchoring agent and the contact interface participate in the coordinated deformation process of the anchoring system. The interface shear stress concentration is reduced, and its distribution is more uniform through shear deformation inside the bolt. However, if the 
influence of shear deformation is ignored, the concentration degree and distribution range of shear stress at the interface will be misestimated during the anchoring design process. The bolt length is short, which results in excessive shear strength at the interface and insufficient strength of the bolt, so the bolt is easily broken down under tensile failure.

\subsection{Influence on bolt stress under actual working conditions}

\section{Stress distribution of interface and bolt}

Surrounding rock deformation, as one of the main factors to be considered, plays an important role in the process of bolt design. The excavation depth and the elastic modulus of surrounding rock determine the supporting parameters of tunnel excavation, which affect the stress distribution of anchorage interface and bolt. The elastic modulus of surrounding rock is taken as $0.5 \mathrm{GPa}, 2 \mathrm{GPa}$ and $3.5 \mathrm{GPa}$ respectively, and the insitu stress conditions as $5 \mathrm{MPa}, 10 \mathrm{MPa}$ and $15 \mathrm{MPa}$ respectively. The stress distributions of interface and bolt are shown in Fig. 10 and Fig. 11.

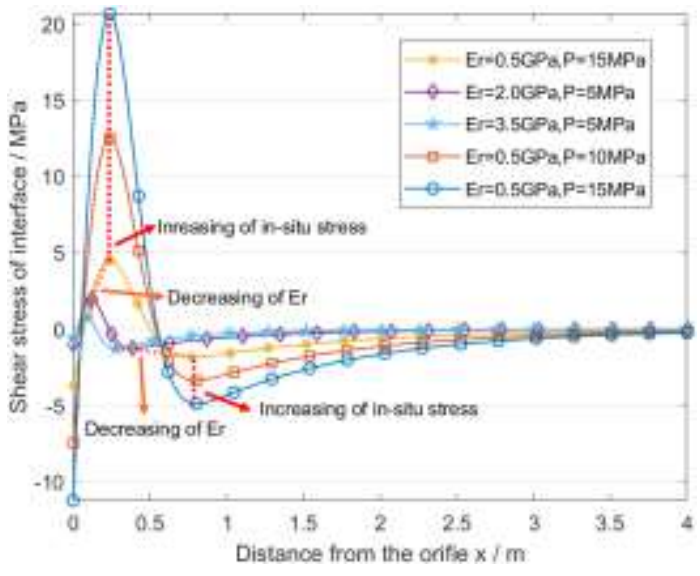

Fig.10 Distribution of shear stress at interface under different deformation conditions of surrounding rock

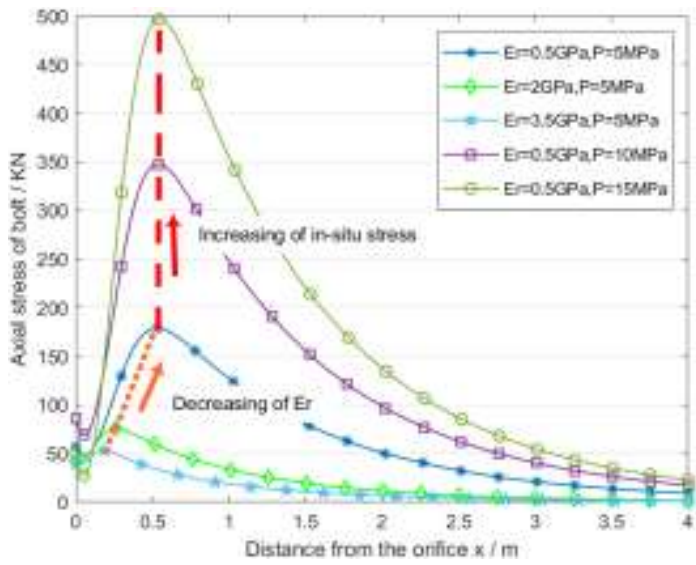

Fig.11 Distribution of axial force of bolt under different deformation conditions of the surrounding rock

By comparing the stress distribution law of interface and bolt under different in-situ stress and elastic modulus of surrounding rock, it can be known that:

1) Under the actual working condition, ensuring the shear stress strength of the pick-up length and bolt tensile strength of the neutral point is the key to controlling the anchoring stability. Under different surrounding rock conditions, the distribution of interface shear stress is basically the same. There is always a "neutral point" which divides the bolt into pick-up length and anchor length, and the first peak shear stress of interface in pick-up length is always greater than the second one in anchor length. Because of the deformation of the surrounding rock, the anchorage force mechanism evolves from passive to active support, which makes the axial force distribution of the bolt quite different from that of the peak axial force in the orifice under the drawing condition. As the interface shear stress on both sides of the neutral point is in the opposite direction, the axial force of the bolt reaches the maximum at the neutral point. Thus, the neutral point part is the most prone to break down. In the bolt design, the stability at the neutral point should be particularly checked if the strength of extension meets the requirements.

2) The elastic modulus of surrounding rock has different effects on the interface shear stress and the bolt axial force, and the maximum positive shear stress is affected more significantly. When the in-situ stress is 5 $\mathrm{MPa}$, with the decrease of the elastic modulus of the surrounding rock, the peak shear stress of interface and the bolt axial force gradually increases. The peak shear stress gradually moves to the depth of the bolt, while the peak axial force always stays at the neutral point. When the elastic modulus of surrounding rock changes from 3.5 $\mathrm{GPa}$ to $2 \mathrm{GPa}$ and $0.5 \mathrm{GPa}$, the maximum positive shear stress increases by $160 \%$ and $128 \%$, respectively, while the maximum negative shear stress increases by $0.96 \%$ and $47.3 \%$, respectively. The peak axial force increases by $41 \%$ and $136 \%$, respectively. It can be seen 
that the influence of maximum positive shear stress is gradually weakened while the maximum negative shear stress and the bolt axial force is gradually increased with the decreasing of the elastic modulus of surrounding rock. Thus, in a deep soft rock tunnel, the property of the surrounding rock is the key factor to control the deformation and stability behaviors of the tunnel.

3) The distribution law of interface shear stress and bolt axial force is basically consistent with that of in-situ stress. Their magnitude will be significantly affected by the changes of in-situ stress. With the increase of in-situ stress, the interface shear stress and the axial force of the bolt increase obviously, while the change of the peak stress position isn't obvious. When the in-situ stress increases from $5 \mathrm{MPa}$ to $15 \mathrm{MPa}$, the maximum positive shear stress increases by $178 \%$ and $64.7 \%$, while the maximum negative shear stress increases by $83.1 \%$ and $45.5 \%$, and the axial force increases by $94.4 \%$ and $42.9 \%$, respectively. Thus, the influence of peak stress of interface and bolt decreases gradually with the increase of in-situ stress, which indicates the necessity of anchor support during the excavation of a deep tunnel.

4) Surrounding rock deformation also has a significant impact on the failure of the bolt. When the deformation of the surrounding rock is large, the maximum positive shear stress is much larger than the maximum negative shear stress, and the shear stress of the contact interface is obviously concentrated in the pick-up length. The interface stress condition is so poor that the shear failure is easy to occur. With the decrease of surrounding rock deformation, the difference between the maximum positive shear stress and the maximum negative shear stress is reduced. The distribution of shear stress at the contact interface is gradually uniform. With the improved interface stress condition, the shear failure of the interface is relatively difficult to occur, and the extension strength of the bolt becomes the key to control anchoring stability. Thus, in order to ensure the long-term stability of the anchorage system, both the shear strength of interface and the extension strength of the bolt should be considered comprehensively in deep soft rock engineering.

\section{The neutral point and the optimal anchor} length
According to the distribution law of interface shear stress under different surrounding rock conditions, the position of the neutral point almost does not change with the in-situ stress, but it is affected by the elastic modulus of surrounding rock. As the elastic modulus of the surrounding rock decreases, the position of the neutral point gradually moves to the depth of the bolt under the influence of prestressing and deformation of the surrounding rock. Therefore, the higher elastic modulus of surrounding rock means the closer neutral point to the orifice, which is consistent with the result of Tetsuro ESAKI [14]. The position comparison of the neutral point between Tetsuro [14] and the model proposed in this paper is listed in the table. 1. The position of the neutral point proposed in this paper is close to the result of Tetsuro [14] when the elastic modulus of the surrounding rock is low. While the depth of the neutral point is smaller than that of the result of the Tetsuro [14] with the increase of elastic modulus of surrounding rock. That is mainly because the model proposed in this paper takes the anchoring agent as a separate transfer medium to establish a relationship among surrounding rock, anchoring agent and bolt, while Tetsuro ESAKI considers the bolt and anchoring agent as a kind of uniform medium. When the elastic modulus of the surrounding rock is low, the deformation of the surrounding rock is large. The bolt and anchoring agent can be regarded as a kind of uniform medium because the deformation of the anchoring agent is relatively small compared with the surrounding rock. However, as the elastic modulus of surrounding rock increases, the relative deformation of the anchoring agent cannot be ignored due to the decrease of surrounding rock deformation. Therefore, under the influence of interface shear stress caused by the deformation of surrounding rock, the neutral point will move towards the orifice without considering the deformation of the anchoring agent.

Table 1 Influence of rock properties on bolt parameters $(\mathrm{P}=5$

MPa)

\begin{tabular}{ccccc}
\hline Modulus of & recommended & Neutral & Neutral & \\
elasticity & bolt & point in & point of & Pick-up \\
$/ \mathrm{GPa}$ & Length $/ \mathrm{m}$ & this & Tetsuro & ratio $/ \%$ \\
\hline
\end{tabular}




\begin{tabular}{ccccc}
\hline & & paper/m & ESAKI & \\
\hline 0.5 & 4.0 & 0.51 & 0.68 & 12.75 \\
2 & 2.5 & 0.23 & 0.67 & 9.7 \\
3.5 & 2.0 & 0.15 & 0.62 & 8 \\
\hline
\end{tabular}

The recommended bolt length and the pick-up ratio of pick-up length to bolt length are shown in the table. 1. When the elastic modulus of surrounding rock changes from $0.5 \mathrm{GPa}$ to $3.5 \mathrm{GPa}$, the pick-up ratio decreases slightly with the decrease of effective anchorage length, which indicates that the neutral point and the effective anchorage length change synchronously under different lithologic conditions. Considering the safety stock, the pick-up ratio can be set as $20 \%$. Therefore, during the design process of the bolt, the pick-up length can be estimated based on the shear stress position of the interface by the field drawing experiment first. Then the optimal length of the bolt can be determined according to the pick-up ratio. Taking the in-situ stress of $5 \mathrm{MPa}$ as an example, the recommended length of bolt under different surrounding rock conditions is shown in Table 1. The softer surrounding rock means that the longer bolt should be adopted because the shear stress distribution of interface and bolt is large and uneven. While the short bolt should be used when the surrounding rock is hard. Moreover, in soft rock engineering, the long-term stress stability of the bolt should be considered because the stress condition of the bolt is too poor to keep the shear stability of the interface. While in hard rock engineering, the anchor can maintain the stability of excavation as it has better stress conditions.

\section{Discussion}

The influence of surrounding rock deformation is a comprehensive index, which should be consistent with the engineering requirements. In engineering, where the deformation is strictly controlled, the lining with large stiffness is often used to combine with the bolt system. In this case, the influence of surrounding rock deformation can be ignored in the bolt support design because the surrounding rock deformation is very small. However, for some large deformation projects, the yielding supporting technique is often adopted to reduce the stress of the lining structure, where the influence of surrounding rock deformation cannot be ignored. There are great differences in the stress distribution law of full-length bond bolts under different conditions of the surrounding rock. With the increase of tunnel excavation depth, the in-situ stress and the prestress are taken at $5 \mathrm{MPa}$ and $50 \mathrm{KN}$, respectively, and the influence of surrounding rock deformation under different lithological grades [24] is shown in Table 2. The shear stress distribution with and without surrounding rock deformation is shown in Fig 12.

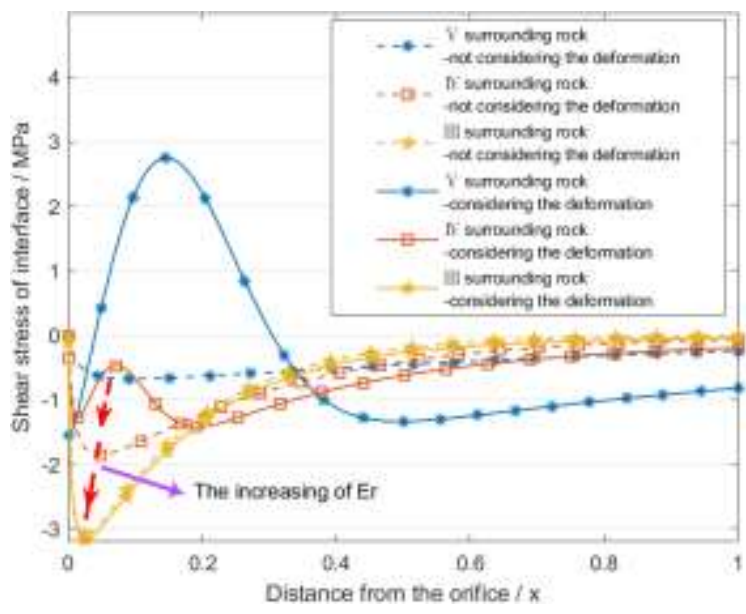

Fig 12 Distribution of shear stress at interface under different surrounding rock grades

Table 2 Influence of different rock grades at interface shear stress

\begin{tabular}{cccc}
\hline $\begin{array}{c}\text { The grade of } \\
\text { surrounding } \\
\text { rock }\end{array}$ & $\begin{array}{c}\text { Modulus elastic } \\
\mathrm{E}_{\mathrm{r}} / \mathrm{GPa}\end{array}$ & $\begin{array}{c}\text { Poisson's } \\
\text { ratio } v\end{array}$ & $\begin{array}{c}\text { The deformation } \\
\text { of surrounding } \\
\text { rock }\end{array}$ \\
\hline III & 15 & 0.25 & $\begin{array}{c}\text { Can be ignored } \\
\text { Should not be } \\
\text { neglected }\end{array}$ \\
IV & 3.5 & 0.32 & $\begin{array}{c}\text { Should not be } \\
\text { neglected }\end{array}$ \\
\hline V & 0.5 & 0.36 &
\end{tabular}

When designing the bolt, whether the deformation of surrounding rock was considered will have a great influence on the analysis of the bolt. Under the condition of $\mathrm{V}$ surrounding rock, when the influence of surrounding rock deformation is significant, the peak shear stress increased by three times compared with that without the influence of surrounding rock deformation. 
The distribution form of shear stress conforms to the neutral point theory. In this case, the influence of surrounding rock deformation should be fully considered instead of simply adopting drawing tests while designing the bolt parameters. As the surrounding rock grade gradually increases to $\mathrm{IV}$, the additional interface shear stress and the bolt axial force from the deformation of surrounding rock gradually decrease, which reduces the differences of the bolt stress with and without surrounding rock deformation gradually. When the grade of surrounding rock increases to III, the influence of surrounding rock deformation can be ignored because the distribution of interface shear stress under the two analysis modes is basically the same. As the grade of surrounding rock increases further, the deformation of surrounding rock can be neglected after excavation and support because it is extremely small in this case. Therefore, under the conditions of IV and V surrounding rock, the deformation of surrounding rock should be considered comprehensively in designing the anchorage parameters. While the grade of surrounding rock is greater than III, the influence of surrounding rock deformation on the bolt stress can be ignored.

\section{Conclusion}

(1) The properties of the contact interface have an important influence on the analysis of bolt stress, including bolt-anchoring agent interface (the first interface) and anchoring agent-surrounding rock interface (the second interface). Based on elastic shear stress-strain theory, the distribution of interface shear stress and axial bolt stress are obtained under drawing and actual working conditions, respectively, with the consideration of the shear deformation of the interface, which provides a theoretical foundation for the design of full-length bond bolt.

(2) Under the drawing condition, the peak shear stress of interface decreases and the distribution range increases under the influence of shear deformation of interface and anchoring agent, which can better describe the behavior of bolt stress under the drawing force. With the increase of elastic modulus of surrounding rock, the peak shear stress of interface increases, and the peak position moves towards the orifice.

(3) Under the actual working condition, the shear stress of the interface has a neutral point near the orifice, and its position is closely related to that of peak shear stress. With the increase of elastic modulus of surrounding rock, the position of the neutral point moves towards the orifice. The best bolt length can be determined by field experiment because the ratio of pick-up length to bolt length varies within 0.2. Compared with the unimodal distribution in drawing conditions, the shear stress of interface changes to bimodal distribution under the influence of surrounding rock deformation. The failure of the bolt always starts from the pick-up length and gradually develops to the depth of the bolt due to the maximum shear stress in the pick-up length.

(4) The properties of the surrounding rock will have a significant influence on the anchorage failure mode. Under the condition of the soft surrounding rock, the distribution range of interface shear stress is large, and the shear stress of pick-up length is significantly greater than that of the anchor length. The longer bolt should be used for support in this case because the bolt is easily pulled out due to the debonding failure of the interface. As the elastic modulus of surrounding rock increases, the distribution range of interface shear stress decreases, and the distribution of interface shear stress tend to be more uniform. The length of bolt could be reduced appropriately because the anchoring failure mode gradually evolves into the tensile failure of the bolt.

\section{References}

[1] Chun-An Y. Mechanical analysis on wholly grouted anchor[J]. Chinese Journal of Rock Mechanics and Engineering, 2000, 19(3): 339-341.

[2] Chun-an Y O U. Theory and application study on stresstransfer mechanism of anchoring system[J]. Chinese Journal of Rock Mechanics and Engineering, 2005, 24(7): $1272-1272$

[3] Cao G J, Jiang H D, Xiong H M. Calculation method of support length for stretched bolts[J]. Chinese Journal of Rock Mechanics and Engineering, 2003, 22(7): 1141-1145.

[4] Yiming Z. Study on mechanical behavior of epoxy bonded 
bolt system and bolt bearing characteristic in coal mine roadway[J]. Journal of China Coal Society, 2012, 37(8): 1423-1424.

[5] He S M, Tian J C, Zhou J T. Study on load transfer of bond prestressed anchor rope[J].Chinese Journal of Rock Mechanics and Engineering, 2006, 25(1): 117-121.

[6] Siming H, Xiaogang Z, Chenghua W. Study on mechanism of prestressed anchoring cable based on modified shear lag model [J]. Chinese Journal of Rock Mechanics and Engineering, 2004, 15.

[7] Farmer I.W.. International Journal of rock Mechanics and Mining Sciences \& Geomechanics Abstracts,1975,12(11).

[8] F. Delhomme,G. Debicki. Numerical modelling of anchor bolts under pullout and relaxation tests[J]. Construction and Building Materials,2009,24(7).

[9] Zhang J, Tang B. Hyperbolic function model to analyze load transfer mechanism on bolts[J]. Chinese Journal of Geotechnical Engineering, 2002, 24(2): 188-192.

[10] Zhong-Xin J. A Gauss curve model on shear stress along anchoring section of anchoring rope of extensional force type [J]. Chinese Journal of Geotechnical Engineering, 2001, 6 .

[11] FREEMAN T J. The behavior of fully-bonded rock bolts in the Kielder experimental tunnel $[\mathrm{J}]$. Tunnels and Tunnelling, 1978, 10(5):37-40.

[12] LI Yingming, ZHAO Chengxing, CONG LI et al. Analysis of stress distribution characteristics of fully anchored bolt based on actual surrounding rock [J].Journal of China Coal Society, 2019,44 (10) : 2966-2973

[13] TAO Z,CHEN J X. Behavior of rock bolting as tunneling support [A]. Stephansson $\mathrm{O}$ ed. Proceedings of the International Symposium on Rock Bolting[C]. Rotterdam:Balkema,1984:87-92.

[14] Tetsuro ESAKI, Yujing JIANG, Yue CAI. Analytical model for grouted rock bolt design of tunnel [J].Japan Society of Civil Engineers,2003,13.

[15] Yue Cai, Tetsuro Esaki,Yujing Jiang. An analytical model to predict axial load in grouted rock bolt for soft rock tunnelling[J].Tunnelling and Underground Space Technology incorporating Trenchless TechnologyResearch, 2004, 12 (6).

[16] Guo-jun W U, Yi-dun C H U, Wei-zhong C. Constitutive model of anchorage interface in underground engineering and its time-effect analysis[J]. Rock and Soil Mechanics,
2011, 32(1): 237-243.

[17] Chong L I, Jin-Hai X U, Ming L I. The mechanical characteristics analysis of fully anchored pre-stressed bolts in coal mines[J]. Journal of Mining and Safety Engineering, 2013, 30(2): 188 .

[18] Mingshu Wang, Xiuren He, Yutian Zheng. Mechanical model of full-length anchoring bolt and its application[J]. Metal mine,1983(04):24-29.

[19] Song Yimin, Deng Linlin, Lv Xiangfeng, et al.Journal of mining and safety engineering,2018,35(06):1122-1128.

[20] Chai J, Zhao W H, Li Y, et al. Pull out tests of fiber Bragg grating sensor fitted bolts[J]. Journal of China University of Mining \& Technology, 2012, 41(5): 719-724.

[21] Shiliang Lu, Lei Tang, Xingan Yang. Bolt anchoring force and anchoring technology[M]. China Coal Industry Publishing Home, Beijing, 1998.

[22] Xiaoqing Wang, Jinghe Yang, Jianzhong Li,et al. Mechanical characteristics analysis of full-length anchoring bolt under typical working conditions considering anchor removal[J/OL]. Journal of china coal society:1-10[202101-19].

[23] Hui W, Yuan-cheng G U O. Debonding analysis of a single anchor using catastrophe theory[J]. Rock and Soil Mechanics, 2016, 37(10): 2833-2838.

[24] JianLing Li. Rock mechanics[M].Chongqing University Press: College civil engineering undergraduate instructional professional standard supporting series of textbooks, 201409.

[25] Xunguo Zhu. Study on anchoring mechanism of grouted rock bolt in underground engineering $[\mathrm{D}]$. Dalian University of Technology, 2007 